\title{
EPSILON NIELSEN FIXED POINT THEORY
}

\author{
ROBERT F. BROWN
}

Received 11 October 2004; Revised 17 May 2005; Accepted 21 July 2005

Let $f: X \rightarrow X$ be a map of a compact, connected Riemannian manifold, with or without boundary. For $\epsilon>0$ sufficiently small, we introduce an $\epsilon$-Nielsen number $N^{\epsilon}(f)$ that is a lower bound for the number of fixed points of all self-maps of $X$ that are $\epsilon$-homotopic to $f$. We prove that there is always a map $g: X \rightarrow X$ that is $\epsilon$-homotopic to $f$ such that $g$ has exactly $N^{\epsilon}(f)$ fixed points. We describe procedures for calculating $N^{\epsilon}(f)$ for maps of 1-manifolds.

Copyright (c) 2006 Robert F. Brown. This is an open access article distributed under the Creative Commons Attribution License, which permits unrestricted use, distribution, and reproduction in any medium, provided the original work is properly cited.

\section{Introduction}

Forster has applied Nielsen fixed point theory to the study of the calculation by computer of multiple solutions of systems of polynomial equations, using a Nielsen number to obtain a lower bound for the number of distinct solutions [4]. Because machine accuracy is finite, the solution procedure requires approximations, but Forster's information is still applicable to the original problem. The reason is that sufficiently close functions on wellbehaved spaces are homotopic and the Nielsen number is a homotopy invariant.

The point of view of numerical analysis concerning accuracy is described by Hildebrand in his classic text [5] in the following way. "Generally the numerical analyst does not strive for exactness. Instead, he attempts to devise a method which will yield an approximation differing from exactness by less than a specified tolerance." The work of Forster does not involve an initially specified tolerance. In particular, although the homotopy between two sufficiently close maps is through maps that are close to both, Forster puts no limitation on the homotopies he employs. The purpose of this paper is to introduce a type of Nielsen fixed point theory that does assume that a specified tolerance for error must be respected.

If distortion is limited to a pre-assigned amount, then it may not be possible, without exceeding the limit, to deform a map $f$ so that it has exactly $N(f)$ fixed points. For a very simple example, consider a map $f: I \rightarrow I=[0,1]$ such that $f(0)=f(2 / 3)=1$ and 
$f(1 / 3)=f(1)=0$. If a map $g$ has $N(f)=1$ fixed point, then there must be some $t \in I$ such that $|f(t)-g(t)|>1 / 3$.

This example suggests a concept of the geometric minimum (fixed point) number of a map $f: X \rightarrow X$ different from the one, $M F[f]$, that is the focus of Nielsen fixed point theory, namely,

$$
M F[f]=\min \{\# \operatorname{Fix}(g): g \text { is homotopic to } f\}
$$

where \# Fix $(g)$ denotes the cardinality of the fixed point set. The distance $d(f, g)$ between maps $f, g: Z \rightarrow X$, where $Z$ is compact and $X$ is a metric space with distance function $d$, is defined by

$$
d(f, g)=\max \{d(f(z), g(z)): z \in Z\}
$$

Given $\epsilon>0$, a homotopy $\left\{h_{t}\right\}: Z \rightarrow X$ is an $\epsilon$-homotopy if $d\left(h_{t}, h_{t^{\prime}}\right)<\epsilon$ for all $t, t^{\prime} \in I$. For a given $\epsilon>0$, we define the $\epsilon$-minimum (fixed point) number $M F^{\epsilon}(f)$ of a map $f: X \rightarrow X$ of a compact metric space by

$$
M F^{\epsilon}(f)=\min \{\#(\operatorname{Fix}(g)): g \text { is } \epsilon \text {-homotopic to } f\} \text {. }
$$

Note that the concept of $\epsilon$-homotopic maps does not give an equivalence relation.

The notation $M F[f]$ for the minimum number incorporates the symbol [ $f$ ], generally used to denote the homotopy class of $f$, because $M F[f]$ is a homotopy invariant. We do not use the corresponding notation for the $\epsilon$-minimum number because it is not invariant on the homotopy class of $f$. For instance, although a constant map $k$ of $I$ is homotopic to the map $f$ of the example, for which $M F^{\epsilon}(f)=3$ for any $\epsilon \leq 1 / 3$, obviously $M F^{\epsilon}(k)=1$ for any choice of $\epsilon$.

Let $f: X \rightarrow X$ be a map of a compact manifold. Just as the Nielsen number $N(f)$ has the property $N(f) \leq M F[f]$, in the next section we will introduce the $\epsilon$-Nielsen number $N^{\epsilon}(f)$, for $\epsilon$ sufficiently small, that has the property $N^{\epsilon}(f) \leq M F^{\epsilon}(f)$. Our main result, proved in Section 3, is a "minimum theorem": given $f: X \rightarrow X$, there exists $g$ with $d(g, f)<\epsilon$ such that $g$ has exactly $N^{\epsilon}(f)$ fixed points. Wecken's minimum theorem, that if $f: X \rightarrow X$ is a map of an $n$-manifold, then there is a map $g$ homotopic to $f$ with exactly $N(f)$ fixed points, requires that $n \neq 2$. It is well known that on all but a few surfaces there are maps $f$ for which no map homotopic to $f$ has only $N(f)$ fixed points, and indeed the gap between $M F[f]$ and $N(f)$ can be made arbitrarily large [2]. In contrast to Wecken's theorem, our result holds for manifolds of all dimensions. Finally, in Section 4, we discuss the problem of calculating $N^{\epsilon}(f)$.

\section{The $\epsilon$-Nielsen number}

Throughout this paper, $X$ is a compact, connected differentiable manifold, possibly with boundary. We introduce a Riemannian metric on $X$ and denote the associated distance function by $d$. If the boundary of $X$ is nonempty, we choose a product metric on a tubular neighborhood of the boundary and then use a partition of unity to extend to a metric for $X$. There is an $\epsilon>0$ small enough so that, if $p, q \in X$ with $d(p, q)<\epsilon$, then there 
is a unique geodesic $c_{p q}$ connecting them. This choice of $\epsilon$ is possible even though the manifold may have a nonempty boundary because the metric is a product on a neighborhood of the boundary. For the rest of this paper, $\epsilon>0$ will always be small enough so that points within a distance of $\epsilon$ are connected by a unique geodesic. We view the geodesic between $p$ and $q$ as a path $c_{p q}(t)$ in $X$ such that $c_{p q}(0)=p$ and $c_{p q}(1)=q$. The function that takes the pair $(p, q)$ to $c_{p q}$ is continuous. If $x \in c_{p q}$ then $d(p, x) \leq d(p, q)$ because $c_{p q}$ is the shortest path from $p$ to $q$ (see [7, Corollary 10.8 on page 62]).

If $f, g: Z \rightarrow X$ are maps with $d(f, g)<\epsilon$, then setting $h_{t}(z)=c_{f(z) g(z)}(t)$ defines an $\epsilon$ homotopy between $f$ and $g$. Thus an equivalent definition of the $\epsilon$-minimum number of $f: X \rightarrow X$ is

$$
M F^{\epsilon}(f)=\min \{\#(\operatorname{Fix}(g)): d(f, g)<\epsilon\} .
$$

For a map $f: X \rightarrow X$, let

$$
\Delta^{\epsilon}(f)=\{x \in X: d(x, f(x))<\epsilon\} .
$$

Theorem 2.1. The set $\Delta^{\epsilon}(f)$ is open in $X$.

Proof. Let $\mathbb{R}^{+}$denote the subspace of $\mathbb{R}$ of non-negative real numbers. Define $D_{f}: X \rightarrow$ $\mathbb{R}^{+}$by $D_{f}(x)=d(x, f(x))$. Since $[0, \epsilon)$ is open in $\mathbb{R}^{+}$, it follows that $\Delta^{\epsilon}(f)=D_{f}^{-1}([0, \epsilon))$ is an open subset of $X$.

For a map $f: X \rightarrow X$, define an equivalence relation on $\operatorname{Fix}(f)$ as follows: $x, y \in \operatorname{Fix}(f)$ are $\epsilon$-equivalent, if there is a path $w: I \rightarrow X$ from $x$ to $y$ such that $d(w, f \circ w)<\epsilon$. The equivalence classes will be called the $\epsilon$-fixed point classes or, more briefly, the $\epsilon$-fpc of $f$.

Theorem 2.2. Fixed points $x, y$ of $f: X \rightarrow X$ are $\epsilon$-equivalent if and only if there is a component of $\Delta^{\epsilon}(f)$ that contains both of them.

Proof. Suppose $x, y \in \operatorname{Fix}(f)$ are $\epsilon$-equivalent and let $w$ be a path in $X$ from $x$ to $y$ such that $d(w, f \circ w)<\epsilon$. Thus, for each $s \in I$ we have $d(w(s), f(w(s)))<\epsilon$ and we see that $w(I) \subset \Delta^{\epsilon}(f)$. Since $w(I)$ is connected it is contained in some component of $\Delta^{\epsilon}(f)$. Conversely, suppose $x, y \in \operatorname{Fix}(f)$ are in the same component of $\Delta^{\epsilon}(f)$. The components of $\Delta^{\epsilon}(f)$ are pathwise connected so there is a path $w$ in it from $x$ to $y$. Since $w$ is in $\Delta^{\epsilon}(f)$, that means $d(w, f \circ w)<\epsilon$ and thus $x$ and $y$ are $\epsilon$-equivalent.

Theorems 2.1 and 2.2 imply that the $\epsilon$-fpc are open in $\operatorname{Fix}(f)$, so there are finitely many of them $\mathbb{F}_{1}^{\epsilon}, \ldots, \mathbb{F}_{r}^{\epsilon}$. We denote the component of $\Delta^{\epsilon}(f)$ that contains $\mathbb{F}_{j}^{\epsilon}$ by $\Delta_{j}^{\epsilon}(f)$. An $\epsilon$-fpc $\mathbb{F}_{j}^{\epsilon}=\operatorname{Fix}(f) \cap \Delta_{j}^{\epsilon}(f)$ is essential if the fixed point index $i\left(f, \Delta_{j}^{\epsilon}(f)\right) \neq 0$. The $\epsilon$-Nielsen number of $f$, denoted by $N^{\epsilon}(f)$, is the number of essential $\epsilon$-fpc.

Theorem 2.3. If fixed points $x$ and $y$ of $f: X \rightarrow X$ are $\epsilon$-equivalent, then $x$ and $y$ are in the same (Nielsen) fixed point class. Therefore each fixed point class is a union of $\epsilon$-fpc and $N^{\epsilon}(f) \geq N(f)$.

Proof. If $x$ and $y$ are $\epsilon$-equivalent by means of a path $w$ between them such that $d(w, f \circ$ $w)<\epsilon$ then $h_{t}(s)=c_{w(s) f(w(s))}(t)$ defines a homotopy, relative to the endpoints, between $w$ and $f \circ w$ so $x$ and $y$ are in the same fixed point class. Therefore a fixed point class 
$\mathbb{F}$ of $f$ is the union of $\epsilon$-fpcs. If $\mathbb{F}$ is essential, the additivity property of the fixed point index implies that at least one of the $\epsilon$-fpc it contains must be an essential $\epsilon$-fpc. Thus $N^{\epsilon}(f) \geq N(f)$.

The $\epsilon$-Nielsen number is a local Nielsen number in the sense of [3], specifically $N^{\epsilon}(f)=n\left(f, \Delta^{\epsilon}(f)\right)$. However, in local Nielsen theory, the domain $U$ of the local Nielsen number $n(f, U)$ is the same for all the maps considered whereas $\Delta^{\epsilon}(f)$ depends on $f$.

Theorem 2.4. Let $f: X \rightarrow X$ be a map, then $N^{\epsilon}(f) \leq M F^{\epsilon}(f)$.

Proof. Given a map $g: X \rightarrow X$ with $d(f, g)<\epsilon$, let $\left\{h_{t}\right\}: X \rightarrow X$ be the $\epsilon$-homotopy with $h_{0}=f$ and $h_{1}=g$ defined by $h_{t}(x)=c_{f(x) g(x)}(t)$. Theorem 2.1 implies that $d(x, f(x)) \geq \epsilon$ for all $x$ in the boundary of $\Delta_{j}^{\epsilon}(f)$. Thus for $x$ in the boundary of $\Delta_{j}^{\epsilon}(f)$ and $t \in I$ we have

$$
d\left(x, h_{t}(x)\right)+d\left(h_{t}(x), f(x)\right) \geq d(x, f(x)) \geq \epsilon
$$

Since $\left\{h_{t}\right\}$ is an $\epsilon$-homotopy, $d\left(h_{t}(x), f(x)\right)=d\left(h_{t}(x), h_{0}(x)\right)<\epsilon$ so $d\left(x, h_{t}(x)\right)>0$, that is, $h_{t}$ has no fixed points on the boundary of $\Delta_{j}^{\epsilon}(f)$. Therefore the homotopy property of the fixed point index implies that

$$
i\left(f, \Delta_{j}^{\epsilon}(f)\right)=i\left(g, \Delta_{j}^{\epsilon}(f)\right) .
$$

Consequently, if $\mathbb{F}_{j}^{\epsilon}=\operatorname{Fix}(f) \cap \Delta_{j}^{\epsilon}(f)$ is an essential $\epsilon$-fpc, then $i\left(g, \Delta_{j}^{\epsilon}(f)\right) \neq 0$ so $g$ has a fixed point in $\Delta_{j}^{\epsilon}(f)$. We conclude that $g$ has at least $N^{\epsilon}(f)$ fixed points.

Although Theorem 2.4 tells us that $N^{\epsilon}(f)$ is a lower bound for the number of fixed points of all maps $g$ that are $\epsilon$-homotopic to $f$, the number $N^{\epsilon}(f)$ is not itself invariant under $\epsilon$-homotopies. In fact it fails to be invariant under $\zeta$-homotopies for $\zeta>0$ arbitrarily small, as the following example demonstrates.

Example 2.5. Let $f: I \rightarrow I$ be the map whose graph is the solid line in Figure 2.1. Let $g: I \rightarrow I$ equal $f$ except on the interval $[p, q]$, where the graph of $g$ is the line segment connecting $(p, f(p))$ and $(q, f(q))$. Given $\zeta>0$, we can adjust $f$ so that setting $h_{t}(x)=$ $\operatorname{tg}(x)+(1-t) f(x)$ for $x \in[p, q]$ and $h_{t}(x)=f(x)=g(x)$ elsewhere defines a $\zeta$-homotopy between $f$ and $g$. However, $N^{\epsilon}(f)=3$ whereas $N^{\epsilon}(g)=1$.

Since $N(f)=1$ for any map of the interval $I$, this example also demonstrates that $3=N^{\epsilon}(f)>N(f)$. For an example where $N^{\epsilon}(f)>N(f)$ in which $N(f)>1$, we consider the map of the circle described by Figure 2.2. (Note: the line in Figure 2.2 labelled $\Gamma_{C}$ is not relevant to the description of the example. However, we will need it in Section 4 for the algorithm that computes $N^{\epsilon}(f)$ for maps of the circle.)

Example 2.6. The circle $S^{1}$ is represented in Figure 2.2 as $I /\{0,1\}$. The map $f$ is of degree -3 so $N(f)=4$. There are a total of seven $\epsilon$-fpc; these consist of single fixed points except for the $\epsilon$-fpc on the left which is essential and the one on the right which is not. Thus we have $N^{\epsilon}(f)=6$. 


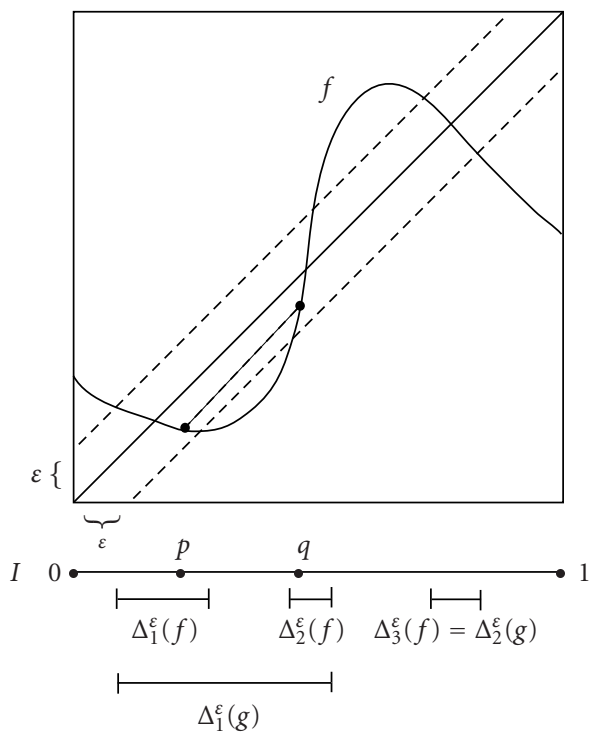

Figure 2.1. Map of the interval.

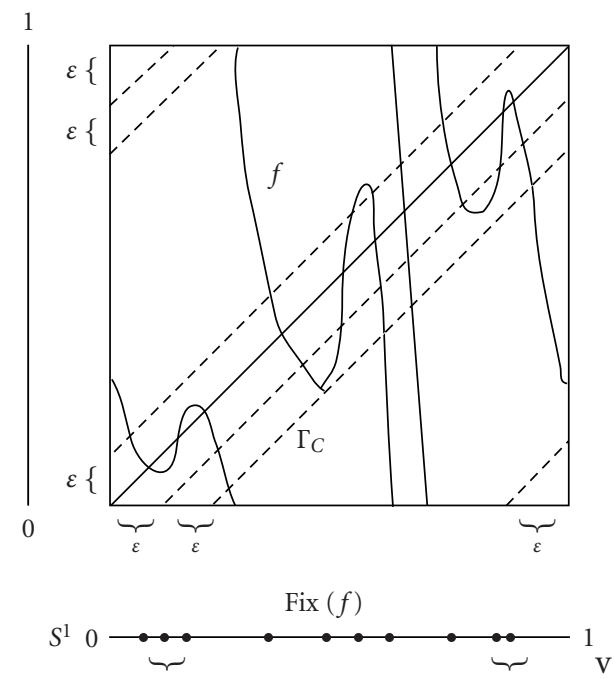

Figure 2.2. Map of the circle.

\section{The minimum theorem}

LeMma 3.1. Let $F$ be a closed subset of a compact manifold $X$ and let $U$ be an open, connected subset of $X$ that contains $F$, then there is an open, connected subset $V$ of $X$ containing $F$ such that the closure of $V$ is contained in $U$. 
Proof. Since $F$ and $X-U$ are disjoint compact sets, there is an open set $W$ containing $F$ such that the closure of $W$ is contained in $U$. There are finitely many components $W_{1}, \ldots, W_{r}$ of $W$ that contain points of the compact set $F$. Let $a_{1}$ be a path in $U$ from $x_{1} \in W_{1} \cap F$ to $x_{2} \in W_{2} \cap F$ and let $A_{1}$ be an open subset of $U$ containing $a_{1}$ such that the closure of $A_{1}$ is in $U$. Since $a_{1}$ is connected, we may assume $A_{1}$ is also connected. Continuing in this manner, we let

$$
V=W_{1} \cup A_{1} \cup W_{2} \cup A_{2} \cup \cdots \cup W_{r},
$$

which is connected. The closures of each of the $W_{i}$ and $A_{i}$ are in $U$ so the closure of $V$ is also in $U$.

Let $\mathbb{F}_{j}^{\epsilon}=\operatorname{Fix}(f) \cap \Delta_{j}^{\epsilon}(f)$ be an $\epsilon$-fpc. By Lemma 3.1, there is an open, connected subset $V_{j}$ of $\Delta_{j}^{\epsilon}(f)$ containing $\mathbb{F}_{j}^{\epsilon}$ whose closure $\mathrm{cl}\left(V_{j}\right)$ is in $\Delta_{j}^{\epsilon}(f)$. For the map $D_{f}: X \rightarrow \mathbb{R}^{+}$ defined by $D_{f}(x)=d(x, f(x))$, we see that $D_{f}\left(\operatorname{cl}\left(V_{j}\right)\right)=\left[0, \delta_{j}\right]$ where $\delta_{j}<\epsilon$. Choose $\alpha_{j}>$ 0 small enough so that $\delta_{j}+2 \alpha_{j}<\epsilon$.

Theorem 3.2 (Minimum Theorem). Given $f: X \rightarrow X$, there exists $g: X \rightarrow X$ with $d(g, f)$ $<\epsilon$ such that $g$ has exactly $N^{\epsilon}(f)$ fixed points.

Proof. We will define $g$ outside $\Delta^{\epsilon}(f)$ to be a simplicial approximation to $f$ such that $d(g, f)<\alpha$, where $\alpha$ denotes the minimum of the $\alpha_{j}$. The proof then consists of describing $g$ on each $\Delta_{j}^{\epsilon}(f)$ so, to simplify notation, we will assume for now that $\Delta^{\epsilon}(f)$ is connected and thus we are able to suppress the subscript $j$. Triangulate $X$ and take a subdivision of such small mesh that if $u$ is a simplicial approximation to $f$ with respect to that triangulation, then $d(u, f)<\alpha / 2$ and, for $\sigma$ a simplex that intersects $X-\operatorname{int}(V)$, we have $u(\sigma) \cap \sigma=\varnothing$. By the Hopf construction, we may modify $u$, moving no point more than $\alpha / 2$, so that it has finitely many fixed points, each of which lies in a maximal simplex in $V$ and therefore in the interior of $X$ (see [1, Theorem 2 on page 118]). We will still call the modified map $u$, so we now have a map $u$ with finitely many fixed points and it has the property that $d(u, f)<\alpha$.

Refine the triangulation of $X$ so that the fixed points of $u$ are vertices. Since $V$ is a connected $n$-manifold, we may connect the fixed points of $u$ by paths in $V$, let $P$ be the union of all these paths. With respect to a sufficiently fine subdivision of the triangulation of $X$, the star neighborhood $S(P)$ of $P$, which is a finite, connected polyhedron, has the property that the derived neighborhood of $S(P)$ lies in $V$. Let $T$ be a spanning tree for the finite connected graph that is the 1-skeleton of $S(P)$, then $T$ contains $\operatorname{Fix}(u)$. Let $R(T)$ be a regular neighborhood of $T$ in $V \cap \operatorname{int}(X)$ then, since $T$ is collapsible, $R(T)$ is an $n$-ball by [8, Corollary 3.27 on page 41]. Thus we have a subset $W=\operatorname{int}(R(T))$ of $V$ containing $\operatorname{Fix}(u)$ and a homeomorphism $\phi: W \rightarrow \mathbb{R}^{n}$. We may assume that $\phi(\operatorname{Fix}(u))$ lies in the interior of the unit ball in $\mathbb{R}^{n}$, which we denote by $B_{1}$. Set $\phi^{-1}\left(B_{1}\right)=B_{1}^{*}$. If $x \in B_{1}^{*}$, then

$$
d(x, u(x)) \leq d(x, f(x))+d(f(x), u(x))<\delta+\alpha<\epsilon
$$

so there is a unique geodesic $c_{x u(x)}$ connecting $x$ to $u(x)$. Consider the map $H: B_{1}^{*} \times I \rightarrow$ $X$ defined by $H(x, t)=c_{x u(x)}(t)$, then $H^{-1}(W)$ is an open subset of $B_{1}^{*} \times I$ containing $B_{1}^{*} \times\{0\}$. Therefore, there exists $t_{0}>0$ such that $H\left(B_{1}^{*} \times\left[0, t_{0}\right]\right) \subset W$. 
Denote the origin in $\mathbb{R}^{n}$ by $\mathbf{0}$ and let $\mathbf{0}^{*}=\phi^{-1}(\mathbf{0})$. Define a retraction $\rho: B_{1}^{*}-\mathbf{0}^{*} \rightarrow$ $\partial B_{1}^{*}$, the boundary of $B_{1}^{*}$, by

$$
\rho(x)=\phi^{-1}\left(\frac{1}{|\phi(x)|} \phi(x)\right) .
$$

Define $K: B_{1}^{*} \times\left[0, t_{0}\right] \rightarrow W$ by setting $K\left(\mathbf{0}^{*}, t\right)=\mathbf{0}^{*}$ for all $t$ and, otherwise, let

$$
K(x, t)=\phi^{-1}(|\phi(x)| \phi(H(\rho(x), t))) .
$$

The function $K$ is continuous because $\phi\left(H\left(\partial B_{1}^{*} \times I\right)\right)$ is a bounded subset of $\mathbb{R}^{n}$. Now define $D_{K}: B_{1}^{*} \times\left[0, t_{0}\right] \rightarrow \mathbb{R}^{+}$by $D_{K}(x, t)=d(x, K(x, t))$. Since $D_{K}^{-1}([0, \eta))$ is an open subset of $B_{1}^{*} \times\left[0, t_{0}\right]$ containing $B_{1}^{*} \times\{0\}$, there exists $0<t_{1}<t_{0}$ such that $d\left(x, K\left(x, t_{1}\right)\right)<\alpha$. Define $v: B_{1}^{*} \rightarrow X$ by $v(x)=K\left(x, t_{1}\right)$.

Next we extend $v$ to the set $B_{2}^{*}$ consisting of $x \in W$ such that $0 \leq|\phi(x)| \leq 2$ by letting

$$
v(x)=c_{x u(x)}\left(\left(1-t_{1}\right)|\phi(x)|+2 t_{1}-1\right)
$$

when $1 \leq|\phi(x)| \leq 2$. Noting that $v(x)=u(x)$ if $\phi(x)=2$, we extend $v$ to all of $X$ by setting $v=u$ outside $B_{2}^{*}$.

The map $v$ has a single fixed point at $0^{*}$. If $i\left(f, \Delta^{\epsilon}(f)\right) \neq 0$, we let $g=v: X \rightarrow X$. If $i\left(f, \Delta^{\epsilon}(f)\right)=0$, by [1, Theorem 4 on page 123], there is a map $g: X \rightarrow X$, identical to $v$ outside of $B_{1}^{*}$, such that $g$ has no fixed point in $B_{1}^{*}$ and $d(g, v)<\alpha$.

We claim that $d(g, f)<\epsilon$. For $x \notin B_{2}^{*}$, we defined $g(x)=u(x)$ where $d(u, f)<\alpha<\epsilon$. If $x \in B_{2}^{*}-B_{1}^{*}$, then $g(x)=v(x) \in c_{x u(x)}$ so $d(v(x), u(x)) \leq d(x, u(x))$. Therefore,

$$
\begin{aligned}
d(g(x), f(x)) & =d(v(x), f(x)) \\
& \leq d(v(x), u(x))+d(u(x), f(x)) \\
& \leq d(x, u(x))+d(u(x), f(x)) \\
& \leq(d(x, f(x))+d(f(x), u(x)))+d(u(x), f(x)) \\
& <\delta+2 \alpha<\epsilon .
\end{aligned}
$$

Now suppose $x \in B_{1}^{*}$. If $i\left(f, \Delta^{\epsilon}(f)\right) \neq 0$, then $g(x)=v(x)=K\left(x, t_{1}\right)$ so

$$
\begin{aligned}
d(g(x), f(x)) & =d\left(K\left(x, t_{1}\right), f(x)\right) \\
& \leq d\left(K\left(x, t_{1}\right), x\right)+d(x, f(x)) \\
& <\alpha+\delta<\epsilon .
\end{aligned}
$$

If $i\left(f, \Delta^{\epsilon}(f)\right)=0$ then

$$
\begin{aligned}
d(g(x), f(x)) & \leq d(g(x), v(x))+d(v(x), f(x)) \\
& =d(g(x), v(x))+d\left(K\left(x, t_{1}\right), f(x)\right) \\
& \leq \alpha+(\alpha+\delta)<\epsilon
\end{aligned}
$$

which completes the proof that $d(g, f)<\epsilon$. 
We return now to the general case, in which $\Delta^{\epsilon}(f)$ may not be connected. Applying the construction above to each $\Delta_{j}^{\epsilon}(f)$ gives us a map $g: X \rightarrow X$ with exactly $N^{\epsilon}(f)$ fixed points. For $x \notin \Delta^{\epsilon}(f)$ we defined $g$ to be a simplicial approximation with $d(g(x), f(x))<$ $\alpha<\epsilon$. For $x \in \Delta_{j}^{\epsilon}(f)$, the argument just concluded proves that

$$
d(g(x), f(x)) \leq 2 \alpha_{j}+\delta_{j}<\epsilon
$$

because $\alpha$ is the minimum of the $\alpha_{j}$, so we know that $d(g, f)<\epsilon$.

Theorem 3.2 throws some light on the failure of the Wecken property for surfaces [2]. For instance, consider the celebrated example of Jiang [6], of a map $f$ of the pants surface with $N(f)=0$ but $M F[f]=2$. The fixed point set of $f$ consists of three points, one of them of index zero. The other two fixed points, $y_{1}$ and $y_{2}$, are of index +1 and -1 respectively and Jiang described a path, call it $\sigma$, from $y_{1}$ to $y_{2}$ such that $\sigma$ is homotopic to $f \circ \sigma$ relative to the endpoints. Suppose $\epsilon>0$ is small enough so that points in the pants surface that are within $\epsilon$ of each other are connected by a unique geodesic. If there were a path $\tau$ from $y_{1}$ to $y_{2}$ such that $\tau$ and $f \circ \tau$ were $\epsilon$-homotopic, then $N^{\epsilon}(f)=0$ and therefore, by Theorem 3.2, there would be a fixed point free map homotopic to $f$. Since Jiang proved that no map homotopic to $f$ can be fixed point free, we conclude that no such path $\tau$ exists. In other words, for any path $\tau$ from $y_{1}$ to $y_{2}$ that is homotopic to $f \circ \tau$ relative to the endpoints, it must be that $d(\tau, f \circ \tau)>\epsilon$.

\section{Calculation of the $\epsilon$-Nielsen number}

In some cases, the $\epsilon$-Nielsen theory does not differ from the usual theory. If a map $f$ : $X \rightarrow X$ has only one fixed point, as a constant map does for example, then there is only one $\epsilon$-fpc so $N^{\epsilon}(f)=N(f)$. For another instance, let $1_{X}: X \rightarrow X$ denote the identity map. Again we have only one $\epsilon$-fpc for any $\epsilon>0$ because $\Delta^{\epsilon}\left(1_{X}\right)=X$.

However, in general we would expect $N^{\epsilon}(f)>N(f)$ and Example 2.5 can easily be modified to produce a map of the interval for which $N^{\epsilon}(f)-N(f)$ is arbitrarily large. The problem of calculating the $\epsilon$-Nielsen number appears to be even more difficult than that for the usual Nielsen number because $N^{\epsilon}(f)$ is not homotopy invariant so it does not seem that the tools of algebraic topology can be applied. The goal then is to obtain enough information from the given map $f$ itself to determine $N^{\epsilon}(f)$. As in the usual Nielsen theory, even a complete description of the fixed point set $\operatorname{Fix}(f)$ is not sufficient, except in extreme cases such as those we noted, without information about the fixed point class structure on $\operatorname{Fix}(f)$, which generally has to be obtained in some indirect manner.

We will next present a procedure that determines $N^{\epsilon}(f)$ for a map $f: I \rightarrow I$ just by solving equations involving the map $f$ itself. Let $A^{\epsilon}$ denote the set of solutions to the equation $f(x)=x+\epsilon$ and let $B^{\epsilon}$ be the set of solutions to $f(x)=x-\epsilon$. Thus, looking back at Figure 2.1, $A^{\epsilon}$ corresponds to the intersection of the graph of $f$ and the boundary of the $\epsilon$-neighborhood of the diagonal that lies above the diagonal and $B^{\epsilon}$ corresponds to the intersection of the graph of $f$ and the boundary of that neighborhood that lies below the diagonal. The set $Q^{\epsilon}=I-\left(A^{\epsilon} \cup B^{\epsilon}\right)$ is a union of intervals that are open in I. We define the essential intervals in $Q^{\epsilon}$ to be the intervals with one endpoint in $A^{\epsilon}$ and 
the other in $B^{\epsilon}$ together with the interval $[0, x)$ if $x \in B^{\epsilon}$ and $(x, 1]$ if $x \in A^{\epsilon}$. Although $Q^{\epsilon}$ may consist of infinitely many intervals, only finitely many of them can be essential. Otherwise, let $A_{0}^{\epsilon} \subseteq A^{\epsilon}$ be the endpoints of the essential intervals, then $A_{0}^{\epsilon}$ contains a sequence converging to some point $a_{0} \in A^{\epsilon}$. Thus every neighborhood of $a_{0}$ contains points of $A_{0}^{\epsilon}$, but it also contains points of $B^{\epsilon}$, which contradicts the continuity of $f$. The reason is that, by the definition of essential interval, for any set of three successive points $a_{1}<a_{2}<a_{3}$ in $A_{0}^{\epsilon}$ there must be at least one $b \in B^{\epsilon}$ such that $a_{1}<b<a_{3}$.

We claim that $N^{\epsilon}(f)$ equals the number of essential intervals. Note that $\Delta^{\epsilon}(f)$ is a union of intervals of $Q^{\epsilon}$. For $J$ an interval of $Q^{\epsilon}$, we write its closure as $\operatorname{cl}(J)=\left[j_{0}, j_{1}\right]$. If $J$ is an essential interval, then one of the points $\left(j_{0}, f\left(j_{0}\right)\right)$ and $\left(j_{1}, f\left(j_{1}\right)\right)$ must lie above the diagonal and the other below it, and therefore $f$ has a fixed point in $J$. The graph of $f$ restricted to $\mathrm{cl}(J)$ can be deformed vertically, keeping the endpoints fixed, to the line segment connecting $\left(j_{0}, f\left(j_{0}\right)\right)$ and $\left(j_{1}, f\left(j_{1}\right)\right)$ so, by the homotopy property of the fixed point index, $i(f, J)= \pm 1$. Now let $K$ be an interval of $Q^{\epsilon}$ that is not essential and write its closure as $\mathrm{cl}(K)=\left[k_{0}, k_{1}\right]$. Either both of $\left(k_{0}, f\left(k_{0}\right)\right)$ and $\left(k_{1}, f\left(k_{1}\right)\right)$ lie above the diagonal or both lie below it and thus the restriction of the graph of $f$ to $\mathrm{cl}(K)$ can be deformed vertically, keeping the endpoints fixed, to the line segment connecting $\left(k_{0}, f\left(k_{0}\right)\right)$ and $\left(k_{1}, f\left(k_{1}\right)\right)$. Since the components of the complement of the diagonal in $I \times I$ are convex, the line segment does not intersect the diagonal and therefore $i(f, K)=0$. We have proved that the essential intervals in $Q^{\epsilon} \subset I$ are the $\Delta_{j}^{\epsilon}$ that contain the essential $\epsilon$-fpc of $f$ and that establishes our claim.

For an example of the use of this procedure, we return to Example 2.5, pictured in Figure 2.1. Denoting points of $I$ that lie in $A^{\epsilon}$ by $a$ and those in $B^{\epsilon}$ by $b$ then, in the ordering of $I$ we have

$$
0<(a<b)<(b<a)<(a<b)<1 .
$$

We note that there are three essential intervals, as indicated by the parentheses, so again we have $N^{\epsilon}(f)=3$.

A modification of the previous procedure can be used for maps $f: S^{1} \rightarrow S^{1}$. In this case, the set of points $(x, y) \in S^{1} \times S^{1}$ such that $d(x, y)=\epsilon$ is the union of two disjoint simple closed curves, which we will call $\Gamma_{A}$ and $\Gamma_{B}$, on the torus. We denote by $A^{\epsilon} \subset S^{1}$ the points $x$ such that $(x, f(x)) \in \Gamma_{A}$ and by $B^{\epsilon}$ the points $x \in S^{1}$ such that $(x, f(x)) \in \Gamma_{B}$. Since the complement of the diagonal in $S^{1} \times S^{1}$ is connected, if an interval in $S^{1}-\left(A^{\epsilon} \cup B^{\epsilon}\right)$ has one endpoint in each of those sets, it does not necessarily contain a fixed point of $f$. Thus, in order to identify intervals of that type that do contain fixed points, we consider the set of points $(x, y) \in S^{1} \times S^{1}$ such that $d(x, y)=2 \epsilon$. This set is the union of two disjoint simple closed curves and we choose one of them arbitrarily, calling it $\Gamma_{C}$ (see Figure 2.2). Denote by $C^{\epsilon} \subset S^{1}$ the points $x$ such that $(x, f(x)) \in \Gamma_{C}$. The set $Q^{\epsilon}=S^{1}-\left(A^{\epsilon} \cup B^{\epsilon} \cup C^{\epsilon}\right)$ is a union of connected open subsets of $S^{1}$ which we will refer to as open intervals in $S^{1}$. Now we may call an open interval essential if one of its endpoints is in $A^{\epsilon}$ and the other in $B^{\epsilon}$. Again, there are only finitely many essential intervals and the number of them equals $N^{\epsilon}(f)$. The reasoning is similar to that for maps of the interval. An essential interval $J$ does contain fixed points and a homotopy shows that $i(f, J)= \pm 1$. If an interval $K$ in $S^{1}$ is not essential, that means either that at least one of the endpoints of $K$ lies in $C^{\epsilon}$ or both 
its endpoints lie in one of the sets $A^{\epsilon}$ or $B^{\epsilon}$. Then there is a homotopy of $f$ to a map that is identical to $f$ outside of $K$ but has no fixed points in $K$, so we conclude that $i(f, K)=0$.

Referring to Figure 2.2 for Example 2.6, we can write

$$
\begin{aligned}
(1=) 0 & <(a<b)<c<(a<b)<(b<a)<(a<b) \\
& <c<(a<b)<c<(a<b)<b<b<c<1(=0)
\end{aligned}
$$

and conclude that $N^{\epsilon}(f)=6$.

\section{Acknowledgments}

I thank Robert Greene for geometric advice. I am also grateful to the referee whose conscientious review lead to significant improvements in this paper.

\section{References}

[1] R. F. Brown, The Lefschetz Fixed Point Theorem, Scott, Foresman, Illionis, 1971.

[2] _ Nielsen fixed point theory on manifolds, Nielsen Theory and Reidemeister Torsion (Warsaw, 1996), Banach Center Publ., vol. 49, Polish Academy of Sciences, Warsaw, 1999, pp. 19-27.

[3] E. Fadell and S. Husseini, Local fixed point index theory for non-simply-connected manifolds, Illinois Journal of Mathematics 25 (1981), no. 4, 673-699.

[4] W. Forster, Computing "all" solutions of systems of polynomial equations by simplicial fixed point algorithms, The Computation and Modelling of Economic Equilibria (Tilburg, 1985) (D. Talman and G. van der Laan, eds.), Contrib. Econom. Anal., vol. 167, North-Holland, Amsterdam, 1987, pp. 39-57.

[5] F. B. Hildebrand, Introduction to Numerical Analysis, 2nd ed., International Series in Pure and Applied Mathematics, McGraw-Hill, New York, 1974.

[6] B. Jiang, Fixed points and braids, Inventiones Mathematicae 75 (1984), no. 1, 69-74.

[7] J. Milnor, Morse Theory, Annals of Mathematics Studies, no. 51, Princeton University Press, New Jersey, 1963.

[8] C. P. Rourke and B. J. Sanderson, Introduction to Piecewise-Linear Topology, Ergebnisse der Mathematik und ihrer Grenzgebiete, vol. 69, Springer, New York, 1972.

Robert F. Brown: Department of Mathematics, University of California, Los Angeles,

CA 90095-1555, USA

E-mail address: rfb@math.ucla.edu 